\title{
Syntheses and Pharmacokinetics Properties of an Iloperidone Pharmaceutical Cocrystal
}

Ting-Ting Zhang a,b, Hai-Tao Wang ${ }^{\text {a,b }}$, Jiang-Tao Jia ${ }^{c}$, Xiao-Qiang Cui ${ }^{\text {a,b, }}{ }^{*}$, Qin Li ${ }^{\text {d }}$, Guang-Shan Zhu ${ }^{\mathrm{c}, \mathrm{d}, * *}$

${ }^{a}$ Department of Materials Science, Key Laboratory of Automobile Materials of MOE, Jilin University, Changchun 130012, PR China

${ }^{b}$ State Key Laboratory of Superhard Materials, Jilin University, Changchun 130012, PR China

${ }^{C}$ State Key Laboratory of Inorganic Synthesis \& Preparative Chemistry, Jilin University, Changchun 130012, PR China

${ }^{d}$ Queensland Micro-and Nanotechnology Centre \$ Environmental Engineering, Griffith Univerisity, Nathan Campus, Brisbane, QLD 4111, Australia

\begin{abstract}
We report here a pharmaceutical cocrystal, which was composed of iloperidone with 3, 5-pyridinedicarboxlic acid by crystal engineering strategy. It was characterized by single crystal X-ray and powder X-ray diffraction, which demonstrated that the cocrystal with high purity was obtained. Pharmacokinetics (PK) study of Jilin University China-Cocrystal 10 (JUC-C10) was performed to evaluate the advantages of this strategy for enhancing the oral absorption of the original active pharmaceutical ingredient (API) of iloperidone. The in vivo study of Beagle dogs revealed that the plasma concentration of JUC-C10 reached the maximum concentration and effective blood level earlier than the original API after oral administration, which suggested that JUC-C10 exhibited a more rapid absorption profile and could thus achieve a rapid onset of action.
\end{abstract}

Keywords Pharmaceutical cocrystal, Single crystal X-Ray diffraction, Power X-Ray diffraction, Pharmacokinetics studies 
The pharmaceutical cocrystals have seen a tremendous increase in the recent 10 years due to the potential physicochemical and biological properties of these materials in such aspects as stability, solubility, dissolution, bioavailability, hygroscopicity and compatibility [1-5]. These compounds are generally constructed from active pharmaceutical ingredients (APIs) $[3,6,7]$ linked by cocrystal formers (CCFs) [2,8-10], which bond to APIs for a synthon via hydrogen bonds or non-covalent bonds $[11,12]$. But beyond that, the CCFs were included not only in their potential applications for bonding but also in the pharmaceutically acceptable formers list, such as Generally Regarded as Safe (GRAS) list and Everything Added to Food in the United States (EAFUS) list [13,14]. In our research, iloperidone, which is a second-generation antipsychotic agent approved by the US Food and Drug Administration (FDA) in 2009 for the treatment of schizophrenia in adults [15], was selected to exploit its novel crystal forms with excellent physicochemical and biological properties. Moreover, iloperidone is classified as a Class II category (low solubility and high permeability) according to the Biopharmaceutics Classification System (BCS) [16], which is practically insoluble in water $(0.012 \mathrm{mg} / \mathrm{mL})$ [17-18]. Oral bioavailability of iloperidone was $<1 \%$ in rat, $5 \%$ in mouse, $19 \%$ in both rabbit and dog, and approximately $36 \%$ in humans. Based on the description, we employed 3 , 5-pyridinedicarboxlic acid to synthesize the cocrystal of iloperidone (Scheme 1).

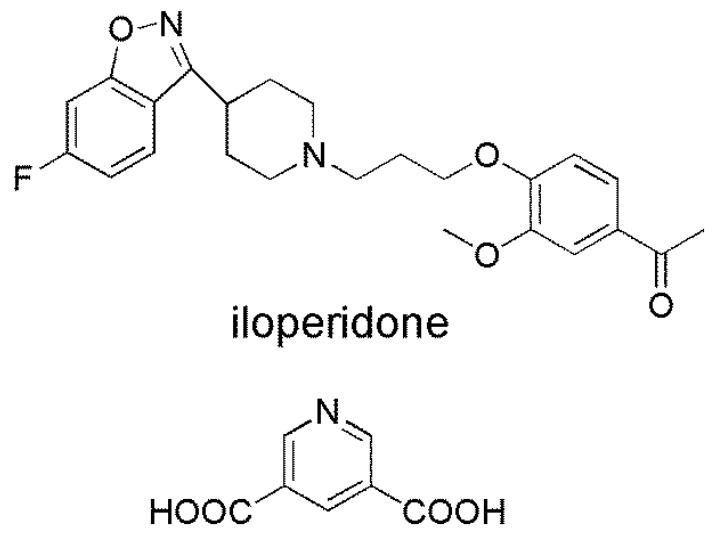

\section{3,5-pyridinedicarboxylic acid}

Scheme 1. Molecular structures of API and CCF.

The crystal X-ray structure determination reveals that the asymmetric unit of JUC-C10 possesses the iloperidone and 3, 5-pyridinedicarboxlic. The parameters of 
JUC-C10 were summarized in Table1. As shown in Fig.1, the carboxylate groups of the 3, 5-pyridinedicarboxlic acid molecule link another 3, 5-pyridinedicarboxlic acid molecule to form a 1D chain on crystallographic b-axis via the hydrogen bonds of $\mathrm{O}-\mathrm{H} \cdots \mathrm{O}$ (Table S1), and the $1 \mathrm{D}$ chain links the $2 \mathrm{D}$ layer via $\pi-\pi$ stacking from the dimers to further generate an infinite $3 \mathrm{D}$ framework. The $\pi-\pi$ stacking dimers are arranged along the crystallographic c-axis and the distance is $3.677 \AA$. In addition, the packing motif is illustrated in head-to-head dimers of the adjacent molecules of iloperidone, and the distance is $3.645 \AA$. The synthesized PXRD pattern of JUC-C10 exhibits good agreement with the calculated XRPD pattern based on a single crystal structure (Fig. 2). EA (\%) calcd for $\mathrm{C}_{31} \mathrm{H}_{32} \mathrm{FN}_{3} \mathrm{O}_{8}$ (Mr 594, JUC-C10): C, 62.7; H, 5.39; N, 7.08. Found: C, 62.6; H, 5.38; N, 7.07.

Table1. Crystallographic Date and structure Refinement Parameters for JUC-C10.

\begin{tabular}{cc}
\hline chemical formula & $\mathrm{C}_{31} \mathrm{H}_{32} \mathrm{FN}_{3} \mathrm{O}_{8}$ \\
formula weight & 593.6 \\
crystal system & Triclinic \\
space group & $\mathrm{P}-1$ \\
$\mathrm{a}(\AA)$ & 9.4398 \\
$\mathrm{~b}(\AA)$ & 9.6102 \\
$\mathrm{c}(\AA)$ & 16.363 \\
$\alpha\left(^{\circ}\right)$ & 89.363 \\
$\beta\left(^{\circ}\right)$ & 78.521 \\
$\gamma\left({ }^{\circ}\right)$ & 74.13 \\
$\mathrm{vol}\left(\AA^{3}\right)$ & 1397.8 \\
$\mathrm{dcal}\left(\mathrm{g} \cdot \mathrm{cm}{ }^{-3}\right)$ & 1.41 \\
$\mathrm{Z}$ & 2 \\
$\mathrm{Nref}$ & 4963 \\
$\mathrm{~T}(\mathrm{~K})$ & 296 \\
$\mathrm{R} 1$ & 0.0449 \\
$\mathrm{wR} 2$ & 0.122 \\
$\mathrm{Gof}$ & 1.117 \\
\hline
\end{tabular}




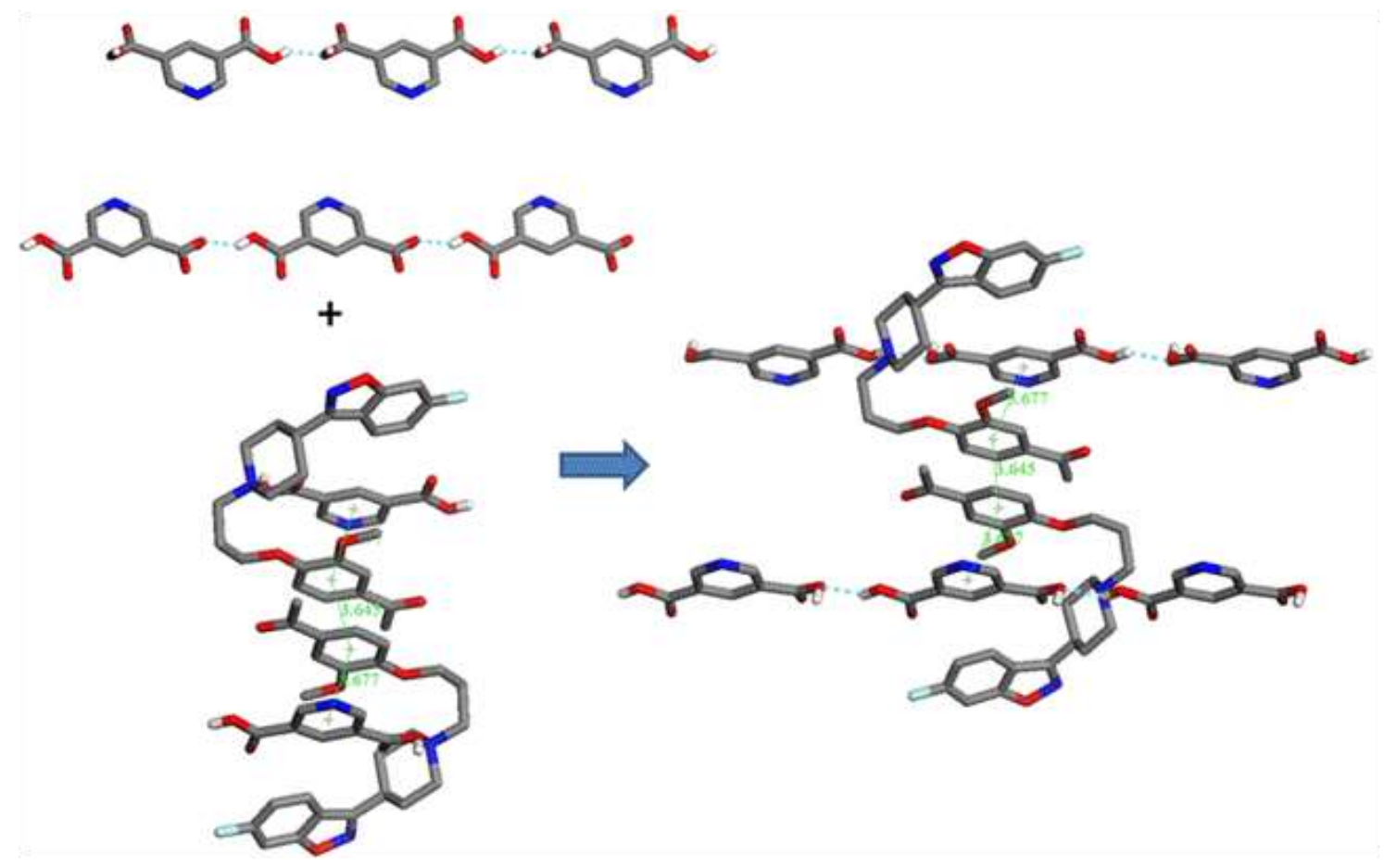

Fig.1. (a) Views of the 1D chain along the crystallographic b-axis. (b) The packing diagram of JUC-C10 shows pairs of 3, 5-pyridinedicarboxlic acid and iloperidone molecules packed to other iloperidone molecules through the $\pi$ - $\pi$ stacking interactions. (c) Schematic representation of a single 3D framework of JUC-C10.

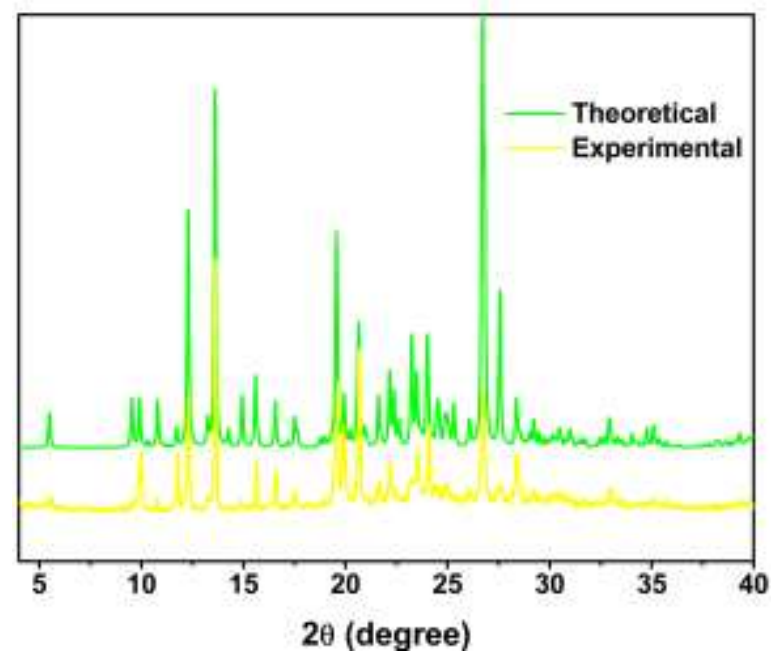

Fig.2. The PXRD patterns of the synthesized (bottom) and the calculated (top).

The PK study of JUC-C10 and the original API was implemented using Beagle dogs, which is the classic model for the evaluation of in vivo absorption. After an overnight fast, six male Beagle dogs were randomly divided into two groups before dosing. In the first group, gelatin capsule containing $4 \mathrm{mg}$ original API was orally administered 
to the first three Beagle dogs. In the second group, the rest of three Beagle dogs were each given a gelatin capsule equivalent to $4 \mathrm{mg}$ iloperidone. Blood samples were collected from jugular vein before oral dosing and at intervals of 30, 45, and $60 \mathrm{~min}$ and $1.5,2,2.5,3,4,6,8,10$, and $12 \mathrm{~h}$ afterwards. Plasma was separated by centrifugation at $3500 \mathrm{rpm}$ for $5 \mathrm{~min}$ and stored at $-20{ }^{\circ} \mathrm{C}$ until $\mathrm{LC}-\mathrm{MS} / \mathrm{MS}$ analysis. Pharmacokinetic parameters were calculated for each subject using Drug and Statistics 3.0 (DAS, China Pharmaceutical University, Nanjing, China) by a non-compartmental model. $\mathrm{C}_{\max }, \mathrm{T}_{\max }, \mathrm{AUC}$ and $\mathrm{t}_{1 / 2}$ could all be obtained. The PK parameters were calculated as follows: iloperidone in original API, $C_{\max } 0.45 \pm 0.01$ $\mathrm{ng} / \mathrm{mL}, \quad \mathrm{t}_{1 / 2} 4.62 \pm 1.09 \mathrm{~h}, \quad \mathrm{~T}_{\max } 4 \pm 0.00 \mathrm{~h}, \mathrm{AUC}_{0}-\infty 2.55 \pm 0.10$ $\mathrm{ng} \cdot \mathrm{h} / \mathrm{mL}$; iloperidone in JUC-C10, $\mathrm{C}_{\max } 0.56 \pm 0.01 \mathrm{ng} / \mathrm{mL}, \mathrm{t}_{1 / 2} 3.01 \pm 1.30 \mathrm{~h}$, $\mathrm{T}_{\max } 3.67 \pm 0.55 \mathrm{~h}, \mathrm{AUC}_{0}-\infty .88 \pm 0.12 \mathrm{ng} \cdot \mathrm{h} / \mathrm{mL}$. As shown in Fig. 3(a), the results indicated that the $\mathrm{AUC}_{0}-\infty$ and $\mathrm{C}_{\max }$ values of JUC-C10 were approximately 1.12-fold and 1.25-fold greater than that of API, respectively. To clarify the relationship of in vivo absorption, the relative in vitro study in this statement was measured by comparing the dissolution rate of original API and JUC-C10. The process of the in vitro study was shown in supplementary materials (Dissolution Study). In Fig. 3(b), although the in vivo absorption behavior of JUC-C10 was improved in PK profile, the in vitro study stated that the dissolution rate of JUC-C10 was not rapid within 180 min compared to the original API from gelatin capsules in simulated gastric fluid. However, in the case of JUC-C10 released from gelatin capsules, reaching $60 \%$ dissolved in the subsequent $300 \mathrm{~min}$, the original API released $23 \%$ without any increase in the later time. This observation suggested that JUC-C10 with an enhanced solubility would be potential to favorably affect the gastrointestinal (GI) absorption and onset action of iloperidone.

The single crystal X-ray and powder X-ray diffraction certified that JUC-C10 with high purity was obtained in a mild condition. After confirming the structures, the dissolution and pharmacokinetic studies were conducted to evaluate the pharmaceutical characteristics of original API and JUC-C10. The great improvement of absorption illustrated that JUC-C10 would be a favored candidate for the 
pharmaceutical industry. In addition, the toxicity and efficacy studies were still underway in our laboratory.

a)

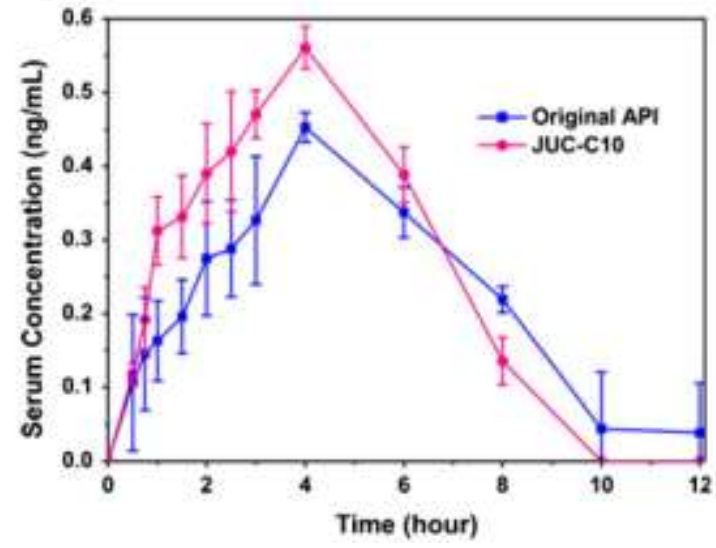

b)

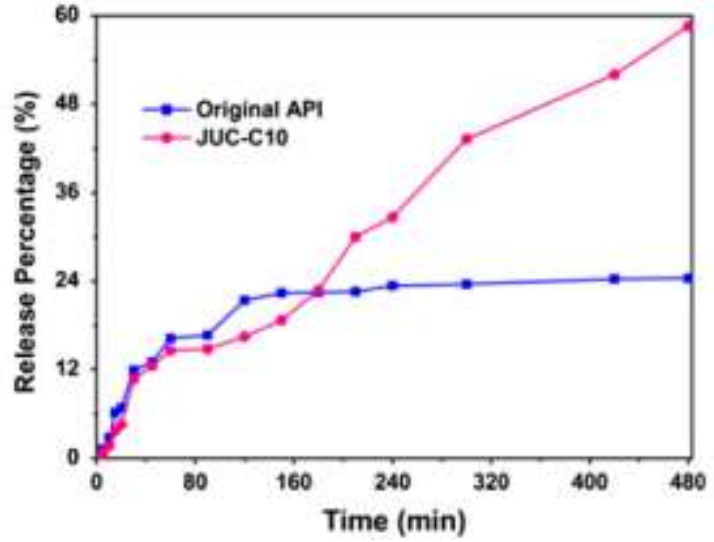

Fig. 3. (a) Mean plasma concentration-time profiles of API and JUC-C10 in Beagle dogs after oral administration. (b) Dissolution profiles in simulated gastric fluid media for the API and JUC-C10.

\section{Acknowledgment}

This work was financially supported by the National Natural Science Foundation of China (No. 21075051 and 21275064), Program for New Century Excellent Talents in University (NCET-10-0433), National Basic Research Program of China (973 Program, no. 2012CB821700), Major International (Regional) Joint Research Project of NSFC (No. 21120102034) and NSFC (No. 20831002).

\section{Appendix A. Supplementary material}

Supplementary data to this article can be found online at http://dx.doi.org/10.1016/j.inoche.2013.11.007.

\section{References}

[1] G.R. Desiraju, Angew. Chem. Int. Ed. 46 (2007) 8342.

[2] Scott G. Fleischman, S. S. K., Jennifer A. McMahon, Brian Moulton, Rosa D. Bailey Walsh, Nai'r Rodri'guez-Hornedo, Michael J. Zaworotko, Cryst. Growth Des. 3 (2003) 909 . 
[3] L.S. Reddy, N.J. Babu, A. Nangia, Chem. Commun. (2006) 1369.

[4] A. Delori, E. Suresh, V.R. Pedireddi, Cryst. Eng. Commun. 15 (2013) 4811.

[5] M. Khan, V. Enkelmann, G. Brunklaus, Cryst. Eng. Commun. 11 (2009) 1001.

[6] David R. Weyna, T. S., Peddy Vishweshwar, Michael J. Zaworotko, Cryst. Growth Des. 9 (2009) 1106.

[7] a.A.N. Nate Schultheiss, Cryst. Growth Des. 9 (2009) 2950.

[8] L. Huang, Adv. Drug Deliv. Rev. 56 (2004) 321.

[9] G. Bolla, P. Sanphui, A. Nangia, Cryst. Growth Des. 13 (2013) 1988.

[10] L. Rajput, P. Sanphui, G.R. Desiraju, Cryst. Growth Des. 13 (2013) 3681.

[11] D.P. McNamara, S.L. Childs, J. Giordano, A. Iarriccio, J. Cassidy, M.S. Shet, R. Mannion, E. O'Donnell, A. Park, Pharm. Res. 23 (2006) 1888.

[12] A. Trask, W. Motherwell, W. Jones, Int. J. Pharm. 320 (2006) 114.

[13] http://www.cfsan.fda.gov/dms/eafus.html.

[14] http://vm.cfsan.fda.gov/dms/eafus.html.

[15] http://www.fda.gov.

[16] G.L. Amidon, H. Lennernas, V.P. Shah, J.R. Crison, Pharm. Res. 12 (1995) 413.

[17] Chemistry reviews. Centre for drug evaluation and research.

[18]http://www.accessdata.fda.gov/drugsatfda_docs/nda/2009/022192s000_ChemR.p df (accessed January 9, 2009). 